\title{
Adenosine stress perfusion CMR in young children: assessment of optimal imaging parameters
}

\author{
Michael J Campbell, Piers Barker, Stephen Darty, Raymond J Kim \\ From 16th Annual SCMR Scientific Sessions \\ San Francisco, CA, USA. 31 January - 3 February 2013
}

\section{Background}

Adenosine stress perfusion CMR is commonly used for assessing coronary artery disease (CAD) in adults. CAD is uncommon in children, but does occur. There is limited experience performing adenosine stress perfusion CMR in young children. Performing stress perfusion CMR in young children presents a number of technical imaging challenges.

Specific Aim: Evaluate image quality and optimal imaging parameters in young children undergoing adenosine stress perfusion CMR.

\section{Methods}

Consecutive patients, who completed clinically ordered CMR adenosine stress perfusion and were $</=5$ yo or $</=25 \mathrm{~kg}$, were enrolled. General anesthesia was utilized in all. All studies were performed on a 1.5T Siemens Avanto system. Adenosine stress perfusion was performed with administration of adenosine $(140 \mu \mathrm{g} / \mathrm{kg} / \mathrm{min})$ for $2-4$ minutes and gadolinium $(0.1 \mathrm{mmol} / \mathrm{kg})$. A prospectively gated saturation recovery turbo flash image was acquired. Imaging parameters were selected by the technician at the time of each scan to optimize image quality. Images were retrospectively reviewed to assess diagnostic quality. The following imaging parameters were recorded: field of view (FOV)-read, FOV-phase, matrix, inversion time, slice thickness and spatial resolution.

\section{Results}

7 patients were enrolled. Demographic information is listed in table 1 . All completed stress perfusion CMR

without adverse events. All had images of diagnostic quality. 2 patients had perfusion defects consistent with inducible ischemia. One underwent coronary angiography (1 lost to follow-up), and had CAD consistent with stress perfusion CMR. One patient with negative stress perfusion CMR underwent coronary angiography and did not have CAD.

Imaging parameters are listed in table 2 . The average FOV-read was $237 \mathrm{~mm}$ (180-300mm), FOV-phase was $175 \mathrm{~mm}(135-239 \mathrm{~mm})$, matrix (frequency) was $198 \mathrm{~mm}(160-300 \mathrm{~mm})$ and matrix (phase) was $146 \mathrm{~mm}$ $(112-139 \mathrm{~mm})$. The average inversion time was $136 \mathrm{msec}$ (100-200 msec). Slice thickness was 7-8mm. The average spatial resolution in both the read and phase direction was $1.2 \mathrm{~mm}(0.9-1.6 \mathrm{~mm})$, and the average voxel size was $1.2 \times 1.2 \times 7.5 \mathrm{~mm}$.

\section{Table 1 Patient Demographics}

\begin{tabular}{|c|c|c|c|}
\hline Patient & $\begin{array}{l}\text { Age } \\
(\mathrm{yr})\end{array}$ & $\begin{array}{l}\text { Weight } \\
(\mathrm{kg})\end{array}$ & Diagnosis \\
\hline 1 & 1 & 8.6 & $\begin{array}{c}\text { Transposition of the great arteries s/p } \\
\text { arterial switch }\end{array}$ \\
\hline 2 & 2 & 14.6 & $\begin{array}{c}\text { Transposition of the great arteries s/p } \\
\text { arterial switch }\end{array}$ \\
\hline 3 & 4 & 22.3 & $\begin{array}{l}\text { Pulmonary atresia/intact ventricular septum } \\
\qquad \mathrm{s} / \mathrm{p} \text { Fontan }\end{array}$ \\
\hline 4 & 5 & 20.7 & $\begin{array}{c}\text { Anomalous origin of left coronary artery from } \\
\text { pulmonary artery s/p repair }\end{array}$ \\
\hline 5 & 5 & 18.5 & $\begin{array}{c}\text { Transposition of the great arteries } s / p \\
\text { arterial switch }\end{array}$ \\
\hline 6 & 5 & 22.5 & $\begin{array}{c}\text { Double outlet right ventricle } s / p \text { ventricular } \\
\text { septal defect closure and pulmonary } \\
\text { valvotomy }\end{array}$ \\
\hline 7 & 5 & 19.4 & $\begin{array}{c}\text { Transposition of the great arteries } s / p \\
\text { arterial switch }\end{array}$ \\
\hline
\end{tabular}

() 
Table 2 Stress Perfusion CMR: Imaging Parameters

\begin{tabular}{ccccccccccc}
\hline Patient & $\begin{array}{c}\text { Age } \\
(\mathrm{yr})\end{array}$ & $\begin{array}{c}\text { Weight } \\
(\mathrm{kg})\end{array}$ & $\begin{array}{c}\text { FOV-read } \\
(\mathrm{mm})\end{array}$ & $\begin{array}{c}\text { FOV-phase } \\
(\mathrm{mm})\end{array}$ & $\begin{array}{c}\text { Matrix- } \\
\text { frequency } \\
(\mathrm{mm})\end{array}$ & $\begin{array}{c}\text { Matrix-read } \\
(\mathrm{mm})\end{array}$ & $\begin{array}{c}\mathrm{Tl} \\
(\mathrm{msec})\end{array}$ & $\begin{array}{c}\text { Slice } \\
\text { thickness } \\
(\mathrm{mm})\end{array}$ & $\begin{array}{c}\text { Spatial resolution- } \\
\text { read }(\mathrm{mm})\end{array}$ & $\begin{array}{c}\text { Spatial resolution- } \\
\text { phase }(\mathrm{mm})\end{array}$ \\
\hline 1 & 1 & 8.6 & 220 & 154 & 160 & 112 & 110 & 8 & 1.4 & 1.4 \\
\hline 2 & 2 & 14.6 & 180 & 135 & 192 & 144 & 180 & 7 & 0.9 & 1.0 \\
\hline 3 & 4 & 22.3 & 300 & 239 & 300 & 239 & 130 & 8 & 1.9 & 1.1 \\
\hline 4 & 5 & 20.7 & 200 & 139 & 176 & 122 & 120 & 8 & 1.1 & 1.1 \\
\hline 5 & 5 & 18.5 & 220 & 150 & 208 & 142 & 200 & 7 & 1.6 & 1.6 \\
\hline 6 & 5 & 22.5 & 260 & 192 & 160 & 118 & 100 & 8 & 1.5 & 1.5 \\
\hline 7 & 5 & 19.4 & 280 & 219 & 192 & 150 & 115 & 8 & & 1.5 \\
\hline
\end{tabular}

FOV=Field of view, $\mathrm{Tl}=$ Inversion time

\section{Conclusions}

CMR adenosine stress perfusion can be performed in young children and images of diagnostic quality acquired. Young children present a number of technical challenges that can be overcome by appropriately adapting imaging parameters to the individual patient size.

\section{Funding}

None.

Published: 30 January 2013

- Convenient online submission

- Thorough peer review

- No space constraints or color figure charges

- Immediate publication on acceptance

- Inclusion in PubMed, CAS, Scopus and Google Scholar

- Research which is freely available for redistribution 